\title{
ERRATUM
}

\section{Electron Probe Microanalysis of Ni Silicides Using Ni-L X-Ray Lines - ERRATUM}

Xavier Llovet, Philippe T. Pinard, Erkki Heikinheimo, Seppo Louhenkilpi, and Silvia Richter

doi:http://dx.doi.org/10.1017/S1431927616011831, Published by Cambridge University Press, 26 October 2016.

A typographical error was published in an equation on p. 8 of "Electron Probe Microanalysis of Ni Silicides Using Ni-L X-Ray Lines" by Llovet et al. (2016), whereby a " 1 " was mistakenly input as a " 0 ". The corrected sentence and equation is published below:

Notice that $\omega_{\mathrm{L} 2}+a_{\mathrm{L} 2}+f_{23}=1$, where $a_{\mathrm{L} 2}$ is the Auger yield for the $\mathrm{L}_{2}$ subshell.

We apologize for this error.

\section{REFERENCE}

Llovet, X., Pinard, P.T., Heikinheimo, E., Louhenkilpi, S. \& Richter, S. (2016). Electron probe microanalysis of Ni silicides using Ni-L X-ray lines. Microsc Microanal 22, doi:http://dx.doi.org/10.1017/S1431927616011831. 